\title{
Response of CFRP Laminates under High Strain Rate Compression until Failure
}

\author{
R.M. Guedes' ${ }^{\text {, M.A. Vaz', F.J. Ferreira }}{ }^{2}$, J. L. Morais ${ }^{3}$ \\ 'Departamento de Engenharia Mecánica e Gestão Industrial, Faculdade de Engenharia, \\ Universidade do Porto, Rua Dr. Roberto Frias s/n, 4200-465 Porto-Portugal \\ ${ }^{2}$ ISEP, Instituto Superior Politécnico, Rua Antönio Bernardino de Almeida 431, 4200-072 Porto, Portugal \\ ${ }^{3}$ Departamento de Engenharias, Universidade de Trás-os-Montes e Alto Douro, \\ Quinta de Prados, 5000-911 Vila Real, Portugal
}

\begin{abstract}
This work describes an experimental characterization: the non-linear rate-dependent mechanical behavior of a composite material under compression. Fiber reinforced polymer matrix composites exhibit non-linear mechanical behavior, except in fiber direction, which is rate-dependent. In this work the Texipreg® HSI60 REM material system was used, comprising high strength carbon fiber and epoxy resin. Unidirectional laminates were tested under uniaxial compression tests on a universal testing machine. The stress/strain curves of several specimens were obtained at three different strain rates of 0.07 , 0.001 and $0.0001 / \mathrm{s}$. In all cases tests were continued until failure was reached to measure the strain rate effect on strength. A 3-parameter constitutive viscoplastic model $/ 6,7 /$ was used to describe the mechanical behavior. This model was developed based on data for strain rate between 0.0001 and $0.07 / \mathrm{s}$. In transverse direction the viscoplastic model was able to predict the high strain rate experiments conducted on a Split Hopkinson Pressure Bar.
\end{abstract}

Key words: High strain rate, Carbon/epoxy, Hopksinson's pressure bar, Constitutive model

\section{INTRODUCTION}

This work is part of a major research project aiming to study the mechanical behavior and failure of CFRP laminates under high strain rate. The increasing use of polymer matrix composite materials in many applications demands the characterization of their mechanical response under a range of strain rates from $10^{-3}$ to $10^{+3} / \mathrm{s}$. Several experimental studies have been performed with the goal of determining the effects of strain rate on the mechanical properties and response of polymer matrix composite systems at high strain rate conditions. A survey done by Gates /1/ describes several analytical models to simulate the rate-dependent response of various types of polymer matrix composites. Later Gates and Sun /2/ devéloped an elasticviscoplastic model for an orthotropic material without tension/compression symmetry and Gates /3/ used an extension of that model to describe an experimental methodology to generate material constants. Goldberg and Stouffer $/ 4 /$ presented an historical survey of experimental development to study the strain rate effect on mechanical properties of polymer matrix composites, followed by constitutive modeling $/ 4,5 /$.

A simple methodology associated to a simple constitutive elastic-viscoplastic model was developed by Sun et al. $/ 6,7 /$. This methodology was applied in the present work to study mechanical behavior of a composite laminate under compression.

\section{THEORETICAL BACKGROUND}

The 3-parameter viscoplastic model used in this work was first presented by Sun et al. 16,7/. This model was developed based on the one-parameter plastic 
model proposed by Chen and Sun [8]. The model was very successful in representing the non-linear behavior of fiber-reinforced polymeric composite materials. The requirements of orthotropic symmetry and assumption that in the fiber direction the material is linear elastic lead to a simple potential function for plane stress state

$$
f=\frac{1}{2} \sigma_{22}^{2}+a_{66} \sigma_{12}^{2}
$$

where $a_{66}$ is an orthotropy coefficient and $\sigma_{22}, \sigma_{12}$ are the transverse and shear stresses, respectively. It is assumed that strain increments are small enough that they can be separated into elastic and plastic parts as

$$
d \varepsilon_{i j}=d \varepsilon_{i j}{ }^{e}+d \varepsilon_{i j}{ }^{p},
$$

where the superscripts $e$ and $p$ denote elasticity and plasticity, respectively. The elastic part follows elastic strain/stress relations for the composite and the plastic strains are obtained from the potential function as

$$
d \varepsilon_{i j}^{p}=d \lambda \frac{\partial f}{\partial \sigma_{i j}}
$$

where $d \lambda$ is the proportional factor. Defining the effective stress as

$$
\bar{\sigma}=\sqrt{3 f}=\sqrt{\frac{3}{2}\left(\sigma_{22}{ }^{2}+a_{66} \sigma_{12}{ }^{2}\right)},
$$

and using the equivalence of plastic work rate $/ 8 /$, the effective plastic strain rate is obtained as

$$
\overline{\dot{\varepsilon}}^{p}=\sqrt{\frac{2}{3}\left(\left(\dot{\varepsilon}_{22}^{p}\right)^{2}+\frac{1}{2 c^{\prime} 66}\left(\dot{\gamma}_{12}^{p}\right)^{2}\right)},
$$

where $\dot{\varepsilon}_{22}{ }^{p}$ and $\dot{\gamma}_{12}{ }^{p}$ are the transverse and shear plastic strain rate, respectively.

Under off-axis constant strain rate, the effective plastic strain can be related with uniaxial applied stress $\sigma_{\mathrm{r}}$ and plastic strain $\varepsilon_{\mathrm{x}}{ }^{\mathrm{p}}$ as

$$
\bar{\sigma}=h(\theta) \sigma_{x},
$$

$$
\bar{\varepsilon}^{p}=\frac{\varepsilon_{x}^{p}}{h(\theta)},
$$

where $h(\theta)$ is an off-axis parameter defined as

$$
h(\theta)=\sqrt{\frac{3}{2}\left(\operatorname{sen}^{4} \theta+2 a_{66} \operatorname{sen}^{2} \theta \cos ^{2} \theta\right)} .
$$

The reference master curve effective plastic strain/effective stress is obtained for the transverse direction, since for this direction $h(\theta)$ is not dependent on the orthotropy coefficient $a_{66}$. The coefficient $a_{66}$ should be such that all curves obtained for all other directions collapse into a single master curve, i.e. the reference master curve. Therefore the viscoplastic model assumes that for each strain rate there is a unique master curve which can be represented by a power law as

$$
\bar{\varepsilon}^{p}=A(\bar{\sigma})^{n}
$$

where $n$ is a material constant and the parameter $A$ is a function of the effective plastic strain rate. Sun et al. 15,6 / proposed the following relationship

$$
A=\chi\left(\left.\right|^{\dot{\varepsilon}} p\right)^{m}
$$

where $\chi$ and $m$ are material constants.

The off-axis material response for the unidirectional loading tests can be predicted using the following nonlinear equation

$$
\dot{\varepsilon}_{x}=\frac{\dot{\sigma}_{x}}{E_{x}}+\dot{\varepsilon}_{x}^{p}
$$

where $E_{\mathrm{x}}$ is the apparent modulus of elasticity of the offaxis specimen which is obtained from the transformation equation

$$
\begin{aligned}
\frac{1}{E_{x}} & =\frac{1}{E_{1}} \cos ^{4} \theta+\left(\frac{1}{G_{12}}-\frac{2 v_{12}}{E_{1}}\right) \sin ^{2} \theta \cos ^{2} \theta \\
& +\frac{1}{E_{2}} \sin ^{4} \theta
\end{aligned},
$$


where $E_{1}, E_{2}, G_{12}$ and $v_{12}$ are the orthotropic elastic constants of the composite. After some algebraic manipulation of Equation (11) a more convenient formulation of the non-linear differential equation can be obtained /6/ using equations (6) and (7) and the following relations

$$
\dot{\varepsilon}_{x}^{p}=\overline{\dot{\varepsilon}}^{p} h(\theta),
$$

and, from equations (9) and (10),

$$
\overline{\dot{\varepsilon}}^{p}=\left(\frac{1}{\chi}\right)^{1 / m}(\bar{\sigma})^{-n / m}\left(\bar{\varepsilon}^{p}\right)^{1 / m}
$$

Finally the non-linear differential equation is obtained

$$
\begin{aligned}
& \dot{\sigma}_{x}+\left(\frac{1}{\chi}\right)^{1 / m} E_{x}[h(\theta)]^{1-n / m-1 / m}\left(\sigma_{x}\right)^{-n / m}\left(\varepsilon_{x}\right)^{1 / m} \\
& -E_{x} \dot{\varepsilon}_{x}=0
\end{aligned}
$$

where

$$
\varepsilon_{x}^{p}=\varepsilon_{x}-\frac{\sigma_{x}}{E_{x}} .
$$

It is interesting to note that the previous equation allows creep analysis. Let us consider the typical creep loading, with a constant load applied $\sigma_{r}=\sigma_{0}$ for $t>0$, and $\varepsilon_{x}=\sigma_{0} / E_{x}$ for $t=0$. Then solving equation (15) for this load condition, the following non-linear creep equation, power law of time, is obtained:

$$
\begin{gathered}
\varepsilon_{x}=\frac{\sigma_{0}}{L_{\mathrm{x}}}+\left[\frac{m-1}{m}\left(\frac{1}{\chi}\right)^{1 / m}[h(\theta)]^{1-n / m-1 / m},\right. \\
\left.\left(\sigma_{0}\right)^{-n / m}\right]{\frac{m}{m-1} i^{m-1}}^{m},
\end{gathered}
$$

where $t$ represents the time.

\section{MATERIALS \& SPECIMEN PREPARATION}

The epoxy pre-preg system used in this work, Texipreg® HSI60 REM manufactured by SEAL, is a modificd epoxy REM reinforced with high strength carbon fiber in the form of unidirectional tape $(0.125$ $\mathrm{mm}$ thick). The laminates were produced on a 40-ton capacity SATIM hot plate press at $130^{\circ} \mathrm{C}$ under 1 bar pressure during 50 minutes and 3 bar pressure during another $60 \mathrm{~min}$, which gives $110 \mathrm{~min}$ of dwell time. The average thickness of cured laminates was $2.5 \mathrm{~mm}$ and fiber content by volume, based on the fiber contents supplied by the pre-preg manufacturer, was $V_{l} \cong 0.60$. From the original manufactured laminates, square samples with $8.5 \mathrm{~mm}$ side were cut using water jet. Since some of the specimens would be tested in the Split Hopkinson Pressure Bar (SHPB), it was essential to obtain an accurate parallelism between the faces that would be in contact with the bars. A specific tool was designed to perform this task. The square specimens were then lapped using fine sandpaper (gril \#600).

\section{EXPERIMENTAL SET-UP}

The low strain rate compression tests were carried out in an INSTRON conventional testing machine in displacement controlled mode with constant cross-head speed of $0.1,1.0$ and $100 \mathrm{~mm} / \mathrm{min}$.

The compression tests at high strain rate were performed with the Split Hopkinson Pressure Bar (SHPB), depicted in Figure 1. The particular setup used in the current study consists of striker, incident and transmission bars made of steel. The bars diameter is 12 $\mathrm{mm}$. The striker bar is $1 \mathrm{~m}$ long, while the incident bar length is $2.5 \mathrm{~m}$ and the transmission bar $1.5 \mathrm{~m}$. The specimen was sandwiched between the incideht bar and the transmission bar. Lubricant grease was applied at the specimen surfaces in contact with the bars to reduce the effect of friction and to provide better contact. Equally, a small amount of lubricant grease was applied at the end of the striker bar to avoid high frequency phenomena in the signal acquired by the oscilloscope, consequence of heterogeneous contact. The full bridge with strain gage transducers $\mathrm{A}$ and $\mathrm{B}$, used as signal monitors, were mounted at 1250 and $215 \mathrm{~mm}$ from the specimen, respectively. The striker bar was released at a pressure of 1 bar by a gas gun specially made for that proposes. The transient strain history is recorded from the strain gages A and B set up on the incident and transmission bars. A PICO CMO0I signal conditioner 
adapter amplifies the gages output signal 10 times. The data is acquired using a LeCroy 9450A digital oscilloscope at a sampling rate of $1 \mathrm{MHz}$. A program named ADAVIDB $/ 9 /$ imports the data from the oscilloscope data storage to the PC for posterior analysis.

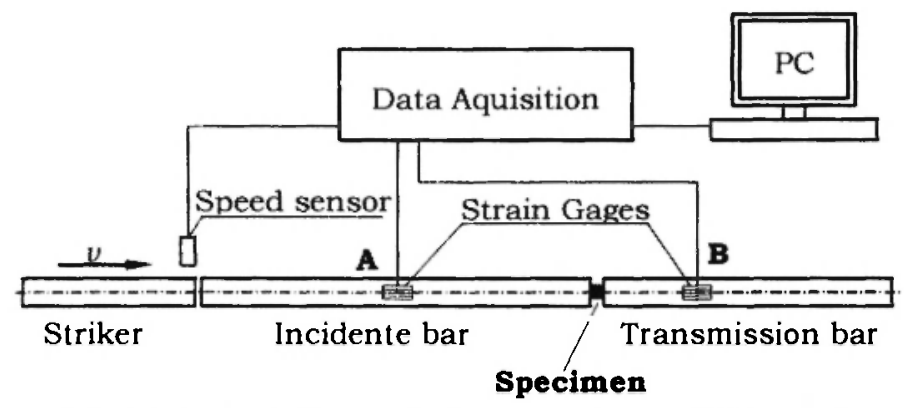

Fig. 1: Schematic representation of the SHPB setup used for compression test.

\section{RESULTS \& DISCUSSION}

An analysis of strain rate dependent mechanical behavior of unidirectional laminates was performed. In Figure 2 the effective stress-effective plastic strain rate is plotted for three strain rates, $0.0001,0.001$ and $0.07 / \mathrm{s}$, for specimens loaded in the transverse direction. In this study, we used the viscoplastic model proposed by Sun et al. $/ 6,7 /$, previously described. In this case the master effective stress -- effective plastic strain was represented as follows:

$$
\bar{\varepsilon}^{p}=A(\bar{\sigma})^{7.00}
$$

where the parameter $A$ is function of the effective plastic strain rate. Assuming that $A$ is a power law function of effective plastic strain, the following relationship was obtained:

$$
A=8.448 \times 10^{-20}\left(\overline{\dot{\varepsilon}}^{p}\right)^{-0.3704}
$$

It was found that the orthotropic parameter $a_{66}$ remains constant $\left(a_{6,}=2.1\right)$ for the three strain rates, $0.0001,0.001$ and $0.07 / \mathrm{s}$. In Figure 2 it is possible to verify the model's ability to reproduce experimental data for the transverse direction.

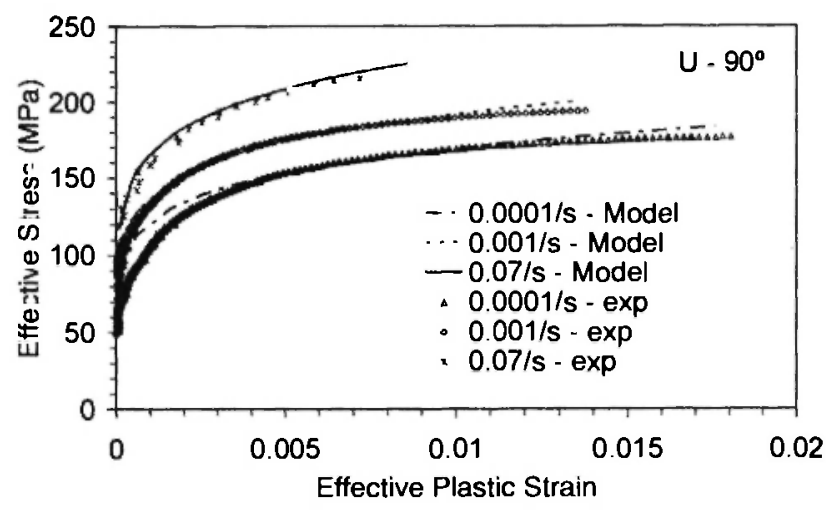

Fig. 2: The effective stress/effective plastic strain curves and model fitted for transverse loading.

In Figures 3, 4, 5 and 6 are depicted model predictions using Equation (15) compared with experimental results at various strain rates for different fiber orientations. For all directions, except $75^{\circ}$ off axis, the model and experimental results are in reasonable agreement. Further, in the case of $75^{\circ}$ off axis, the discrepancy between model and experimental results is only verified in the case of the lower strain rate.

The development of viscoplastic model was based on low strain rates $0.0001,0.001$ and $0.07 / \mathrm{s}$. The experimental tests for high strain rates $400 / \mathrm{s}$ were conducted on a Split Hopkinson Pressure Bar (SHPB) located at the Optical Lab of INEGI. At this moment there are only results for transverse direction. Still, in this direction the viscoplastic model proved to be valid for high strain rates, as depicted in Figure 6.

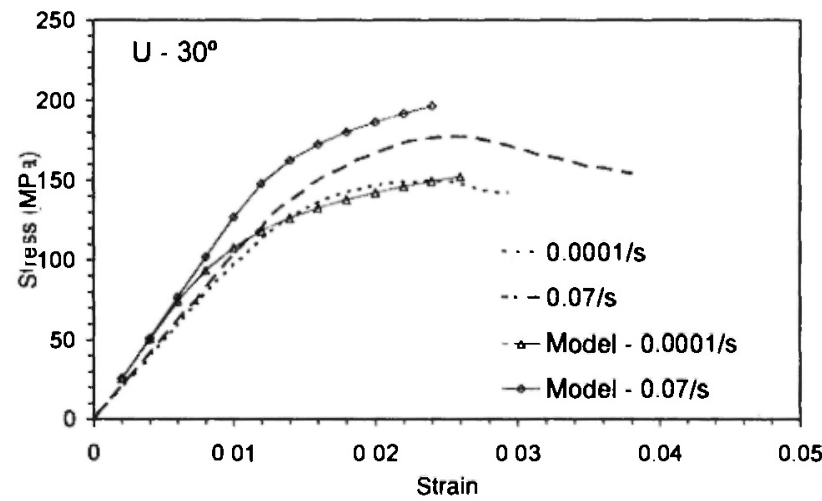

Fig. 3: Comparison of experimental and theoretical stress-strain curves for the $30^{\circ}$ off-axis specimen. 


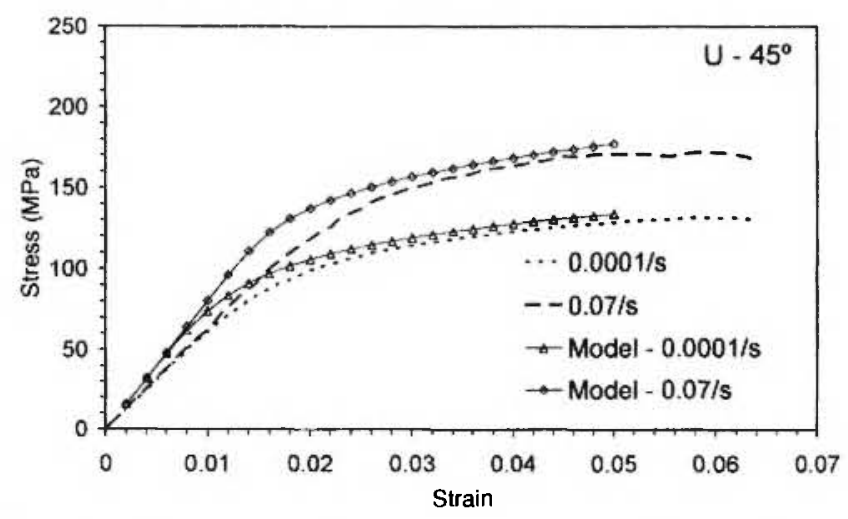

Fig. 4: Comparison of experimental and theoretical stress-strain curves for the $45^{\circ}$ off-axis specimen.

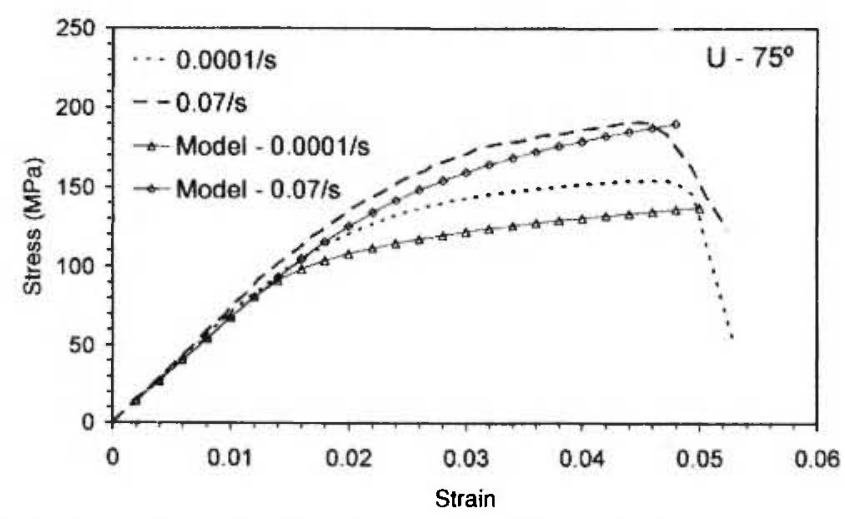

Fig. 5: Comparison of experimental and theoretical stress-strain curves for the $75^{\circ}$ off-axis specimen.

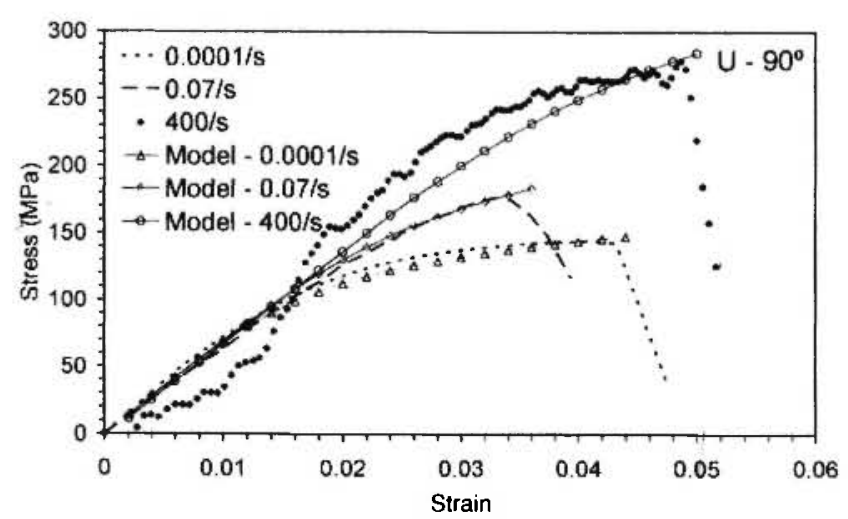

Fig. 6: Comparison of experimental and theoretical stress-strain curves for different strain rates for the transverse loading.

\section{ANALYSIS OF TRANSVERSE FAILURE UNDER IN-PLANE COMPRESSION}

An analysis of failure in the transverse direction was performed. Figure 7 illustrates the shear rupture verified in the $U 90^{\circ}$ laminates. It is possible to see that the two halves have slithered by a defined angle. The total angle measurements average yielded an angle of $60^{\circ} \pm 3.8^{\circ}$. For similar materials Puck and Schürmann $/ 10 /$ measured this fracture angle as being between $\pm 50^{\circ}$ and $\pm 55^{\circ}$.

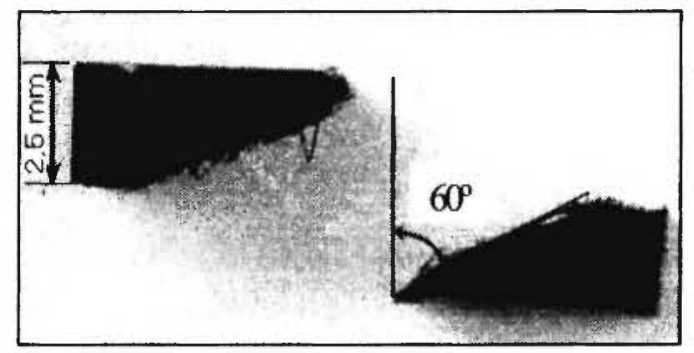

Fig. 7: Shear fracture of the $U 90^{\circ}$ laminates.

One possible approach to model the failure is to relate the time to failure with strain rate. Reifsnider $\boldsymbol{e} t$ al. /11/ verified that the Monkman-Grant classical equation fits data for epoxy at strain rates from quasistatic to very high rate ranges, almost perfectly. For this case the Monkman-Grant equation was determined as,

$$
t_{R}(\dot{\varepsilon})^{0.9983}=0.03861,
$$

where $t_{R}$ represents the time to failure and $\dot{\varepsilon}$ the strain rate. The model extrapolation to larger strain ratios predicts quit well the time to failure for high strain rate as depicted in Figure 8, confirming Reifsnider et al.'s $/ 11 /$ observations. This empirical relation was used as a failure criterion, in conjunction with constitutive law, to predict the strength evolution in function of strain rate. For transverse direction, the prediction obtained was quite close to experimental data, as depicted in Figure 9. 


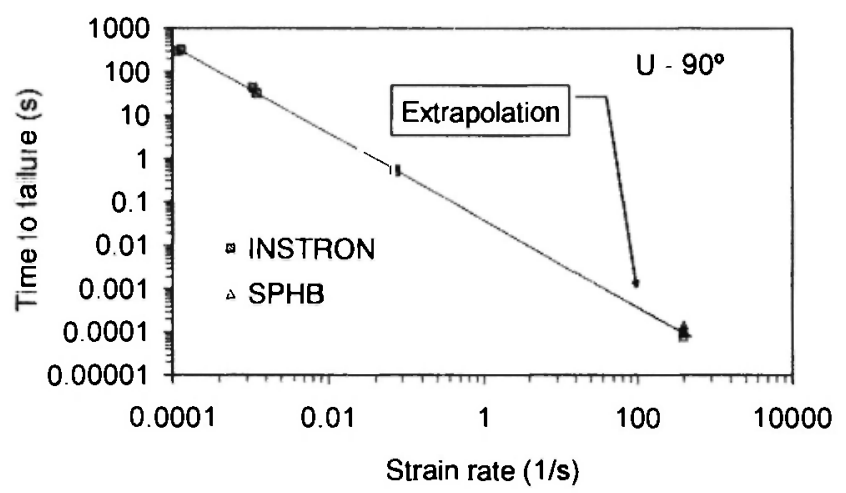

Fig. 8: Strain rate versus time to failure fitted by Monkman-Grant Equation.

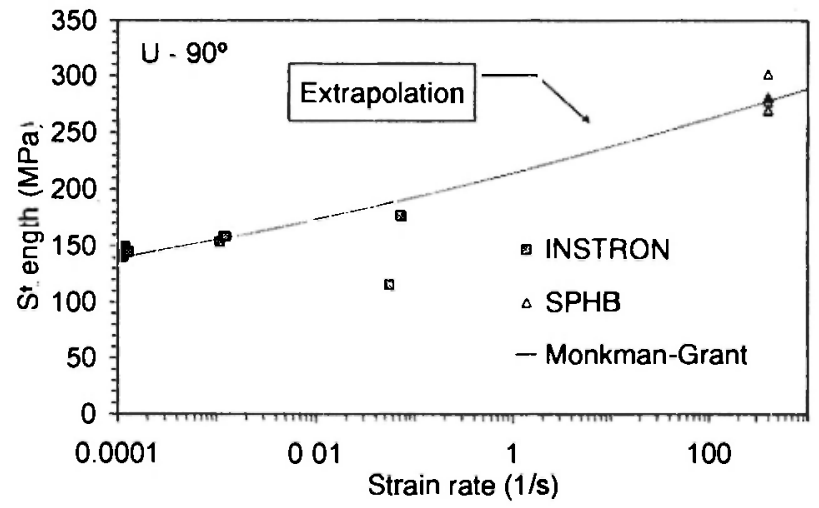

Fig. 9: Experimental and theoretical predictions for strength rate dependency.

\section{CONCLUSIONS}

The rate dependent non-linear behavior of a unidirectional reinforced carbon/epoxy composite under monotonic compression loading was characterized. The 3-parameter constitutive viscoplastic model proposed by Sun et al. $/ 6,7 /$ was used to describe the mechanical behavior. This model was developed based on data for strain rate between 0.0001 and $0.07 / \mathrm{s}$. In transverse direction the viscoplastic model was able to predict the high strain rate experiments conducted on a Split Hopkinson Pressure Bar. Consequently, this 3parameter viscoplastic model demonstrated to be capable to describe the strain rate dependent mechanical behaviour of Texipreg(B) HSI60 REM composite material system, under compression and for a wide range of strain rates, with reasonably accuracy.
In the transverse direction, the strength exhibited a strain rate dependency. The Monkman-Grant relationship was found appropriate to fit and extrapolate failure data from low strain rate to high strain rate. Of course the other way around should also be possible, i.e. extrapolate failure data from high strain rate to low strain rate.

\section{ACKNOWLEDGEMENTS}

This work was funded by Portuguese Science and Technology Foundation (FCT), which is partially supported by the European Community fund FEDER, under Grants POCTI/EME/40048/2001 and POCTI/EME/35901/2000.

\section{REFERENCES}

1. T.S. Gates, "Rate dependent constitutive models for fiber reinforced polymer composites", NASA TM 102665, NASA Langley Research Center, May (1990).

2. T.S. Gates and C.T. Sun, An elastic/viscoplastic constitutive model for fiber reinforced thermoplastic composites, AIAA Journal, 29(3), 457-463 (1991)

3. T.S. Gates, Experimental characterization of nonlinear, rate-dependent behavior in advanced polymer matrix composites, Experimental Mechanics, 32(1), 68-73 (1992)

4. R.K. Goldberg and D.C. Stouffer, "High strain rate deformation modeling of a polymer matrix composite: Part I- Matrix constitutive equations", NASA TM 206969, NASA Lewis Research Center, August (1998).

5. R.K. Goldberg and D.C. Stouffer, Strain rate dependent analysis of a polymer matrix composite utilizing a micromechanics approach, J. Composite Materials, 36(7), 773-793 (2002).

6. S.V. Thiruppukuzhi and C.T. Sun, Models for the strain-rate-dependent behavior of polymer composites, Composites Science \& Technology, 61, 1-12 (2001).

7. J. Tsai and C.T. Sun, Constitutive model for high 
strain rate response of polymeric composites, Composites Science \& Technology, 62, 1289-1297 (2002).

8. C.T. Sun and J.L. Chen, A simple flow rule for characterizing nonlinear behavior of fiber composites, J. Composite Materials, 23, 10091020 (1989).

9. G. Gary and Klepaczko, A Computer Program for the Analysis of SHPB Test, Paris / METZ:1998.
10. A. Puck and H. Schürmann, Failure analysis of FRP laminates by means of physically based phenomenological models, Composite Science \& Technology, 62, 1633-1662 (2002).

11. K. Reifsnider,, C. Mahieux, B. Walther, and F. Sun, Strain rate and temperature effects in polymeric matrices for composite materials, In $\mathrm{A}$. Cardon, H. Fukuda, K. Reifsnider \& G. Verchery (eds.), Recent Developments in Durability Analysis of Composite Materials, 229-236, Rotterdam: Balkema (2000). 
